\title{
Cyclization mechanism of phomopsene synthase: mass spectrometry based analysis of various site-specifically labeled terpenes
}

\author{
Sandip S Shinde ${ }^{1,4}$, Atsushi Minami ${ }^{1}$, Zhi Chen ${ }^{1}$, Tetsuo Tokiwano ${ }^{1}$, Tomonobu Toyomasu ${ }^{2}$, Nobuo Kato ${ }^{3}$, \\ Takeshi Sassa ${ }^{2}$ and Hideaki Oikawa ${ }^{1}$ \\ Elucidation of the cyclization mechanism catalyzed by terpene synthases is important for the rational engineering of terpene \\ cyclases. We developed a chemoenzymatic method for the synthesis of systematically deuterium-labeled geranylgeranyl \\ diphosphate (GGPP), starting from site-specifically deuterium-labeled isopentenyl diphosphates (IPPs) using IPP isomerase \\ and three prenyltransferases. We examined the cyclization mechanism of tetracyclic diterpene phomopsene with phomopsene \\ synthase. A detailed EI-MS analysis of phomopsene labeled at various positions allowed us to propose the structures \\ corresponding to the most intense peaks, and thus elucidate a cyclization mechanism involving double 1,2-alkyl shifts and \\ a 1,2-hydride shift via a dolabelladien-15-yl cation. Our study demonstrated that this newly developed method is highly sensitive \\ and provides sufficient information for a reliable assignment of the structures of fragmented ions.
}

The Journal of Antibiotics (2017) 70, 632-638; doi:10.1038/ja.2017.27; published online 8 March 2017

\section{INTRODUCTION}

Terpene synthases catalyze the cleavage and formation of highly complex multiple bonds via a series of carbocation intermediates to create a significant diversity of terpene molecular skeletons. ${ }^{1}$ Recently, a large number of putative terpene cyclase genes obtained by genomic sequencing and transcriptome analysis were made available through genetic databases of bacteria, fungi and plants. The catalytic mechanism of terpene synthases has been extensively studied using structural biology and examined by quantum mechanics/molecular mechanics calculations. ${ }^{2,3}$ For a rational engineering of terpene cyclases, a detailed elucidation of the reaction mechanism of individual cyclases is essential. Conventionally, whole cell-incorporation experiments of simple isotopically labeled precursors, such as doubly ${ }^{13} \mathrm{C}$ - and ${ }^{2} \mathrm{H}$-labeled sodium acetate and mevalonolactone, are used to study the cyclization pathways. ${ }^{4}$ In vitro analysis of terpene cyclases with specifically isotope-labeled precursors is a more straightforward method for elucidating their mechanisms. In most cases, NMR analysis is used for the correct assignment of the labeling positions. However, owing to the analytical detection limit (usually $>100 \mu \mathrm{g}$ ) and difficulties in the preparation of site-specifically labeled precursors, an alternative method may improve this situation.

EI-MS is one of the most widely used techniques to analyze apolar, volatile, thermostable and low-MW natural products including terpenes, and has been employed for the discovery and characterization of various terpenoids. ${ }^{5}$ More importantly, this analysis requires only small amounts of samples (ca. $10 \mathrm{ng}$ per analysis), thus it is $>100$ times more sensitive than the NMR analysis. In addition, it gives reproducible fragmentations that include structural information on cyclic terpenes, suggesting a potential application of EI-MS in the investigation of the cyclization mechanisms. However, the application of this technique has been hampered by the difficult interpretation of the fragmentation mechanisms. One of the solutions to overcome this issue is the systematic analysis of site-specifically deuterium-labeled samples. Herein, we report the development of a versatile chemoenzymatic method for the synthesis of deuterium-labeled prenyl diphosphates and their study by MS analysis to elucidate the cyclization mechanism of a diterpene synthase, namely phomopsene synthase (PaPS).

Bifunctional terpene synthases, which possess a prenyltransferase domain at the $\mathrm{N}$-terminal and a terpene cyclase domain at the $C$-terminal, represent a new family of terpene synthases. In contrast to other terpene synthases, this type of enzymes can synthesize diterpenes or sesterterpenes owing to their ability to convert cellularly abundant farnesyl diphosphate (FPP) to geranylgeranyl diphosphate (GGPP) or geranylfarnesyl diphosphate as well as cyclize the resultant prenyl diphosphate. In 2007, Sassa and co-workers identified the first bifunctional terpene synthase PaFS, which gives fusicoccadiene possessing a tricyclic 5-8-5 hydrocarbon skeleton. ${ }^{6}$ Following this pioneering work, a homology-based cloning allowed us to identify homologous enzymes such as PaPS, ophiobolin F synthase and sesterfisherol synthase. $^{7-9}$ The cyclization products of the growing

${ }^{1}$ Division of Chemistry, Graduate School of Science, Hokkaido University, Sapporo, Japan; ${ }^{2}$ Department of Bioresource Engineering, Yamagata University, Yamagata, Japan and

${ }^{3}$ The Institute of Scientific and Industrial Research, Osaka University, Osaka, Japan

${ }^{4}$ Current address: Division of Organic, CSIR-National Chemical Laboratory, Pune 411008, India.

Correspondence: Professor H Oikawa, Department of Chemistry, Graduate School of Science, Hokkaido University, Hokkaido, Sapporo 060-0810, Japan.

E-mail: hoik@sci.hokudai.ac.jp

This paper is dedicated to Professor Satoshi Omura, 2015 Nobel Laureate in Physiology or Medicine, in honor of his human health.

Received 23 October 2016; revised 5 January 2017; accepted 27 January 2017; published online 8 March 2017 
a

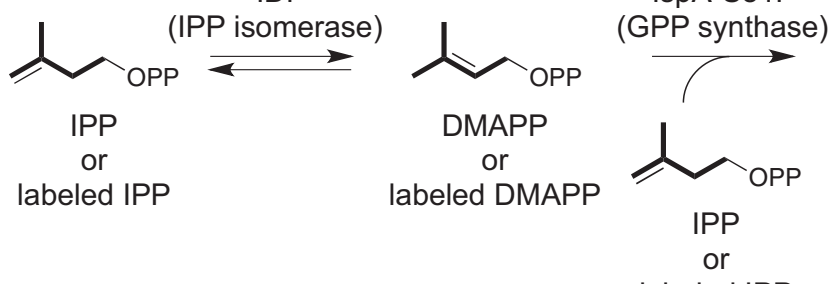

labeled IPP

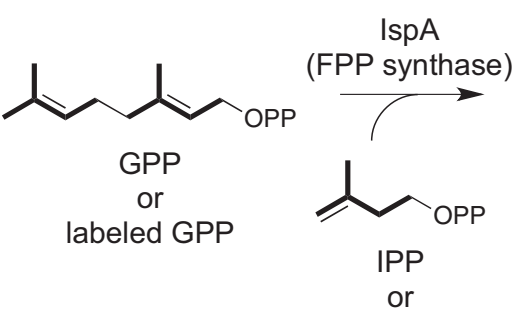

labeled IPP

PaPS

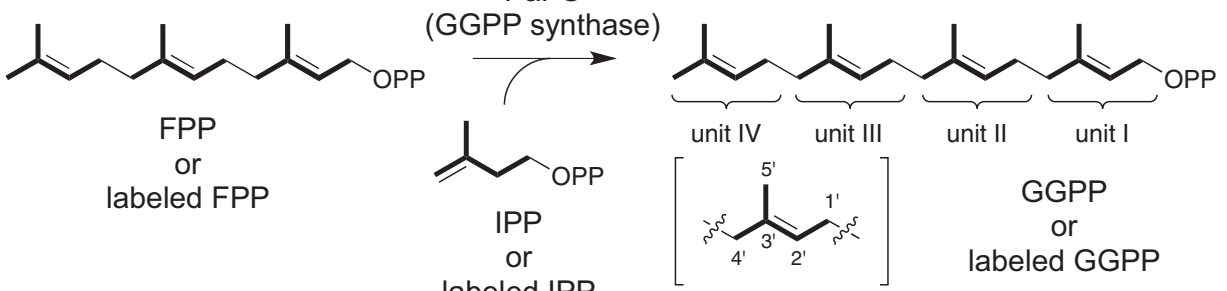

labeled IPP

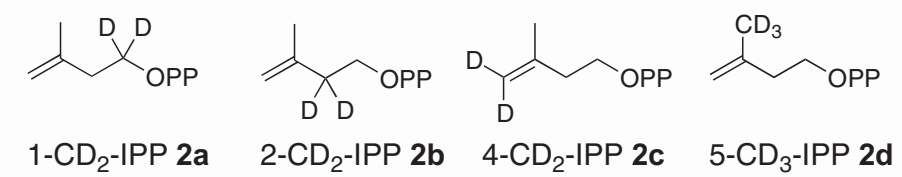

b

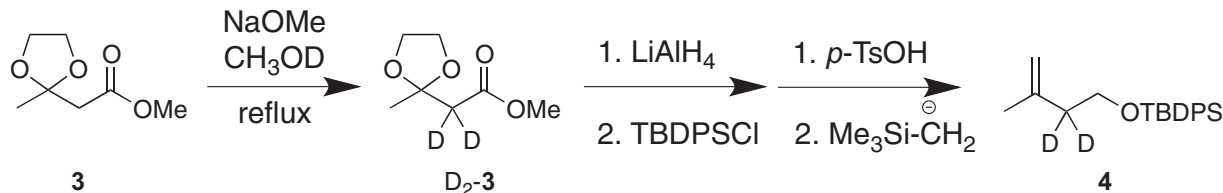

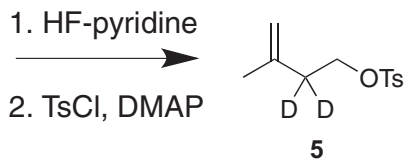

$\left(\mathrm{Bu}_{4} \mathrm{~N}\right)_{3} \mathrm{P}_{2} \mathrm{O}_{7} \mathrm{H}$<smiles>[2H]C(=O)P[PbH2]</smiles>

$2-C D_{2}-$ IPP $2 b$

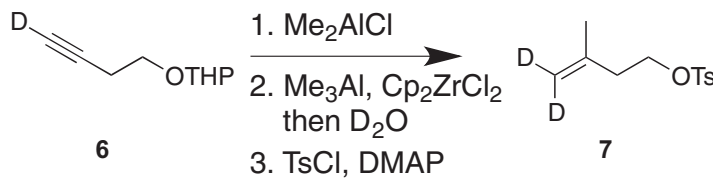

d

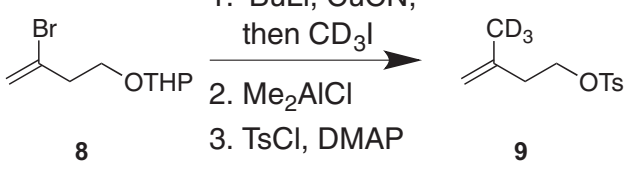

Scheme 1 (a) Outline of the chemoenzymatic synthesis of deuterium labeled 1 . Synthetic schemes of (b) 2-CD2-IPP 2 b, (c) $4-C_{2} D_{2}-I P P 2 c$ and (d) $5-C D_{3}-I P P 2 d$.

family of terpene synthases are unique structures featuring polycyclic skeletons such as tricyclic fusicoccadiene/ophiobolin $\mathrm{F},{ }^{6,8}$ tetracyclic phomopsene (1)/sesterfisherol ${ }^{7,9}$ and pentacyclic quiannulatene (Scheme 1 and Supplementary Figure S1). ${ }^{10}$ The cyclization can occur under specific control of the terpene synthase, thus it is of particular interest for the researchers in the fields of organic and natural product chemistry. In this study, we applied an MS-based analysis of isotopically labeled products to elucidate the cyclization mechanism of PaPS.

\section{RESULTS AND DISCUSSION}

Chemoenzymatic synthesis of deuterium-labeled prenyl diphosphates

Isopentenyl diphosphate (IPP) is a fundamental building block in the enzymatic synthesis of prenyl diphosphates such as GPP, FPP and
GGPP. ${ }^{1}$ Its isomerization and chain elongation involve four enzymes, that is, IPP isomerase, GPP synthase, FPP synthase and GGPP synthase, and proceed in a highly regio- and stereo-selective manner (Scheme 1a). The function of each of these enzymes has been firmly established by in vitro enzymatic reactions. Therefore, an enzymatic synthesis allows us to prepare a series of site-specifically deuterated prenyl diphosphates from a limited set of site-specifically labeled IPPs (2a-2d) (Scheme 1a).

Initially, $2-\mathrm{CD}_{2}$-IPP $\mathbf{2 b}$ was synthesized as shown in Scheme $1 \mathrm{~b}$. After the base-catalyzed deuterium exchange of protected ester 3 in $\mathrm{CH}_{3} \mathrm{OD}$, the resultant ester $\mathrm{D}_{3}-\mathbf{3}$ was converted into a TBDPS ether via the corresponding alcohol. Ketal deprotection and subsequent Peterson olefination ${ }^{11}$ yielded silyl ether 4 , whose TBDPS protecting group was then converted into a tosyl group 5 before the following phosphorylation step. 4-CD $-\mathrm{CD}_{2}$ IPP $2 \mathrm{c}$ was synthesized starting from 
a

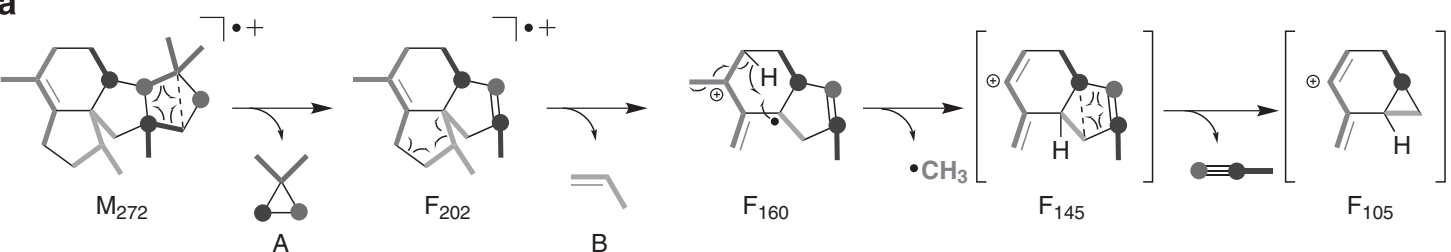

b

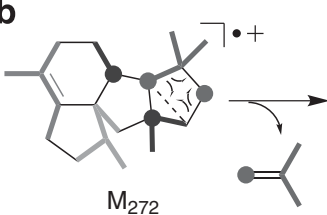

D
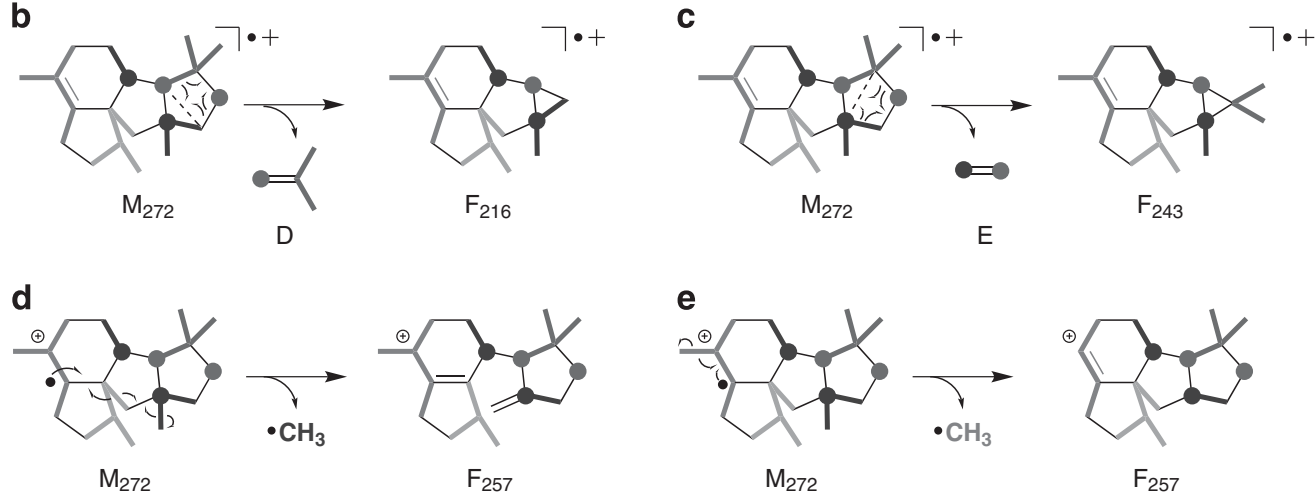

$\mathrm{F}_{257}$

$\mathrm{M}_{272}$

$\mathrm{F}_{257}$

f
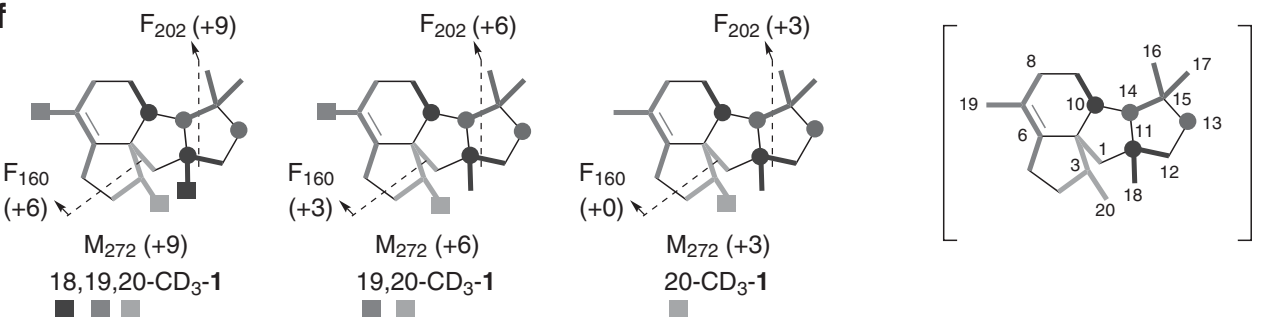

20

Figure 1 (a-e) Fragmentation sequences of $\mathbf{1}$. (f) Fragmentations observed in $18,1920-\mathrm{CD}_{3}-\mathbf{1}, 19,20-\mathrm{CD}_{3}-\mathbf{1}$ and $20-\mathrm{CD}_{3}-\mathbf{1}$. A full colour version of this figure is available at the Journal of Antibiotics journal online.

deuterium-labeled alkyne 6. After deprotection, the regioselective carboalumination ${ }^{12}$ of the resultant alcohol with $\mathrm{Me}_{3} \mathrm{Al}$ and $\mathrm{Cp}_{2} \mathrm{ZrCl}_{2}$ and subsequent quenching with $\mathrm{D}_{2} \mathrm{O}$ gave the desired alcohol, which was converted into tosylate 7 (Scheme 1c). For the synthesis of $5^{\prime}-\mathrm{CD}_{3}$-IPP 2d, the vinyl anion prepared from THP-protected vinyl bromide $\mathbf{8}$ upon treatment with $t$-BuLi followed by $\mathrm{CuCN}$ was coupled with $\mathrm{CD}_{3} \mathrm{I}$ to give THP ether, which was converted into the corresponding tosylate 9 (Scheme 1d). Each tosylate was subjected to phosphorylation using a standard protocol. ${ }^{13}$ Together with 1- $\mathrm{CD}_{2}$-IPP 2a, which was synthesized according to a literature procedure, ${ }^{13,14}$ we prepared a total of four specifically deuteriumlabeled IPP 2a-2d.

To facilitate the discussion on the labeling positions in both GGPP precursor and phomopsene 1, the prenyl chain of GGPP was divided into four isoprene units I-IV, and the corresponding positions were highlighted with the same color (Scheme 1a). In order to perform the chemoenzymatic synthesis of various $\left[{ }^{2} \mathrm{H}\right]$-GGPPs, we prepared IPP isomerase (IDI), ${ }^{15}$ GPP synthase (IspA-S81F), ${ }^{16}$ FPP synthase (IspA) ${ }^{17}$ and PaPS ${ }^{7}$ according to literature procedures. Although the PT domain of PaPS was able to convert dimethylallyl diphosphate (DMAPP) and GPP into GGPP, the reaction was too sluggish. ${ }^{6,7}$ Thus, IspA was added to achieve a rapid conversion of the prenyl diphosphate precursors.

The incubation of $\mathbf{2} \mathbf{a}$ and non-labeled DMAPP with IspA-S81F gave specifically labeled $\left[{ }^{2} \mathrm{H}\right]-\mathrm{GPP}$, which was then incubated with IspA and PaPS to give site-specifically labeled $\left[{ }^{2} \mathrm{H}\right]-1$ via III-[1-CD 2$]-G G P P$
(Supplementary Scheme S1). Similarly, using the three remaining $\left[{ }^{2} \mathrm{H}\right]$-IPPs $\mathbf{2 b}-\mathbf{2 d}$ as extender units, we prepared three specifically labeled $\left[{ }^{2} \mathrm{H}\right]$-GPPs which were converted into isoprene unit III-specifically labeled $\left[{ }^{2} \mathrm{H}\right]-\mathbf{1}$ via the corresponding III- $\left[{ }^{2} \mathrm{H}\right]-\mathrm{GGPP}$. For the preparation of the starter unit DMAPP, $\left[{ }^{2} \mathrm{H}\right]-\mathbf{2 a}-\mathbf{2 d}$ were isomerized with IPP isomerase. After dilution with non-labeled IPP to suppress the incorporation of labeled IPP, the resultant DMAPP was converted into isoprene unit IV-specifically labeled $\mathbf{1}$ via IV- $\left[{ }^{2} \mathrm{H}\right]-$ GGPPs with IspA and PaPS (Supplementary Scheme S1). Thus, we developed an efficient method to synthesize all combinations of specifically deuterium-labeled GGPP and the corresponding cyclization product $\mathbf{1}$ by conversion of simple starting units $\mathbf{2 a - 2 d}$ with prenyltransferases and/or IPP isomerase.

\section{MS-based analysis of site-specifically labeled terpenes}

In the EI-MS spectrum of $\mathbf{1}$, we observed the following major fragment ions at $\mathrm{m} / \mathrm{z} 257\left(\mathrm{~F}_{257}\right), 202\left(\mathrm{~F}_{202}\right), 160\left(\mathrm{~F}_{160}\right)$ and $145\left(\mathrm{~F}_{145}\right)$ (Supplementary Figure S2a), ${ }^{7}$ as well as two other fragment ions $\left(\mathrm{F}_{243}\right.$ and $\left.\mathrm{F}_{216}\right)$. The MS/MS analysis of $\mathrm{F}_{202}$ and $\mathrm{F}_{160}$ showed common product ions at $m / z$ 145, 117 and 105 (Supplementary Figures S2b and c), indicating the fragmentation sequence $\mathrm{M}-\mathrm{F}_{202}-\mathrm{F}_{160}-\mathrm{F}_{145}-\mathrm{F}_{105}$. All these fragments most likely originated from the elimination of a methyl radical and neutral molecules A-E, which have methyl groups. On the basis of these results, we could speculate which structure corresponded to each fragment, as shown Figure 1a. Later, the proposed structures were confirmed by extensive MS analysis of site-specifically labeled 
$\left[{ }^{2} \mathrm{H}\right]-1$. During the MS analysis, we found that fragment $\mathrm{F}_{257}$ consisted of two isomeric fragments, which could be separated during the fragmentation of specifically labeled $\left[{ }^{2} \mathrm{H}\right]-1$ (Figures $1 \mathrm{~d}$,e and Supplementary Figure S3). These fragments may provide further confirmation of the labeling pattern upon detailed HR-MS and MS/ MS analyses. However, these experiments were not performed since sufficient data were obtained from other fragment ions.

In the cyclization with various terpene synthases, most of methyl groups on the isoprene unit of a prenyl diphosphate remain intact in the cyclization product. Thus, we speculated that, in the MS analysis of 1, methyl groups could be useful fragmentation tracers to identify fragments derived from the specific isoprene units I-VI in GGPP. To obtain information on the origin of the methyl groups in the fragments, we analyzed the MS spectra from the enzymatic reactions with multiply and specifically $\left[\mathrm{C}^{2} \mathrm{H}_{3}\right]$-labeled GGPP. The results showed that the labels in the isoprene units I, II, III, IV were located at $\mathrm{C} 20, \mathrm{C} 19, \mathrm{C} 18$ and $\mathrm{C} 16 / \mathrm{C} 17$, respectively (Figure 1f and Supplementary Figure S3).

Considering the proposed structures of the key fragments, we initially focused on the formation of an A-ring that could most likely originate from isoprene units III and IV based on the structure of 1 . Four fragments, $\mathrm{F}_{243}, \mathrm{~F}_{216}, \mathrm{~F}_{202}$ and $\mathrm{F}_{105}$, were useful to examine this mechanism (Figure 2). Mass shifts between non-labeled and labeled-1 enabled us to speculate the labeling pattern of $\mathbf{1}$. Isobutene unit D $\left(\mathrm{M}-\mathrm{F}_{216}\right)$ from IV- $\left[1^{\prime}-\mathrm{CD}_{2}\right]-$ and IV- $\left[5^{\prime}-\mathrm{CD}_{3}\right]-\mathrm{GGPP}$ retained the labels (Figures 2a and b), whereas $\mathrm{C} 5$ unit $\mathrm{A}\left(\mathrm{M}-\mathrm{F}_{202}\right)$ possessed additional labels from III- $\left[4^{\prime}-\mathrm{CD}_{2}\right]$-GGPP (Figure 3c). The neutral C3 unit $\mathrm{C}\left(\mathrm{F}_{202}-\mathrm{F}_{160}-\mathrm{F}_{145}-\mathrm{F}_{105}\right)$ from IV-[2'-CD] and III- $\left[5^{\prime}-\mathrm{CD}_{3}\right]-$ GGPP (Figures $2 \mathrm{~d}$ and e) and ethylene unit $\mathrm{E}\left(\mathrm{M}-\mathrm{F}_{243}\right)$ from IV- $\left[1^{\prime}-\mathrm{CD}_{2}\right]$ - and III- $\left[4^{\prime}-\mathrm{CD}_{2}\right]-\mathrm{GGPP}$ (Figures 2a and c) were eliminated with the labels. Consequently, the origin of all the hydrogen atoms on the A-ring was confirmed, as shown in Figure 2.

Next, we examined the formation of a C/D-ring that originated from isoprene units I and II. Propene unit $\mathrm{B}\left(\mathrm{F}_{202}-\mathrm{F}_{160}\right)$ possessed the labels from I- $\left[2^{\prime}-\mathrm{CD}\right], \quad \mathrm{I}-\left[4^{\prime}-\mathrm{CD}_{2}\right]$ and I- $\left[5^{\prime}-\mathrm{CD}_{3}\right]-\mathrm{GGPP}$ (Figures $2 \mathrm{f}-\mathrm{h})$. By contract, no deuterium label $\left(\mathrm{M}_{272}\right)$ from II-[2'-CD]-GGPP was observed (Figure 2i), while other labels from II- $\left[1^{\prime}-\mathrm{CD}_{2}\right]$, II- $\left[4^{\prime}-\mathrm{CD}_{2}\right]$ and II- $\left[5^{\prime}-\mathrm{CD}_{3}\right]-\mathrm{GGPP}$ were all retained (Supplementary Figure S3). Most of the remaining labels of units I-III were found in $\mathrm{F}_{160}$. An exceptional labeling pattern was observed in the analysis of 1 from III-[2'-CD] and I-[1'-CD $]$-GGPP. Although 1 retained the labels, the expected MS shift of $\mathrm{F}_{202}$ was not observed in these cases. We speculated that an unexpected hydrogen scrambling occurred at these positions. However, this does not influence our proposal for the cyclization mechanism.

For the A-ring formation, labels from unit IV in GGPP located at $\mathrm{C} 13$ and $\mathrm{C} 14-\mathrm{C} 15(\mathrm{C} 17)-\mathrm{C} 16$ in 1, indicated that a C13-C14 bond cleavage occurred during the cyclization. Isoprene unit III C9-C10 and C11(C18)-C12 in 1 was separated by the insertion of C14 between $\mathrm{C} 10$ and $\mathrm{C} 11$. The labeling pattern revealed that two rounds of 1,2-C-shifts are involved in the A-ring construction. In the D-ring construction, the labels from I- $\left[2^{\prime}-\mathrm{CD}\right]$, I- $\left[4^{\prime}-\mathrm{CD}_{2}\right]$ and I- $\left[5^{\prime}-\mathrm{CD}_{3}\right]-$ GGPP were located on the D-ring at C3-C4-C20, respectively, suggesting that the C2-olefinic proton of unit I in GGPP was shifted on $\mathrm{C} 3$ in $\mathbf{1}$. The loss of the label from II-[2'-CD]-GGPP in $\mathbf{1}$ suggested that the final quenching of the $\mathrm{C} 7$-carbocation occurred during the deprotonation at C6 to afford 1. Overall, the cyclization mechanism of $\mathrm{PaPS}$ is summarized in Scheme 2. The cyclization begins with the elimination of the pyrophosphate group of GGPP followed by a cyclization to afford dolabelladien-15-yl cation $\left(\mathbf{1 0}^{+}\right)$. Two sequential 1, 2-alkyl shifts are the key processes accounting for the carbon rearrangement of 1 . The resultant 12 -membered ring cation $11^{+}$then undergoes a C2-C10 cyclization to afford 5-5-9 tricyclic cation $12^{+}$. Subsequent 1, 2-hydride shift and C2-C6 ring closure followed by C6-deprotonation furnish 1.

Sesterterpene mangicol A, derived from a marine fungus, possesses a unique tetracyclic scaffold, which is closely related to $\mathbf{1}$. Its biosynthetic pathway resembles that of 1 and was proposed by feeding experiments of various ${ }^{13} \mathrm{C}$-labeled acetates. ${ }^{18} \mathrm{~A}$ branch point between the metabolic pathways of $\mathbf{1}$ and mangicol is the cyclization of $11^{+} / 11 a^{+}$. Instead of the C2-C10 cyclization in the pathway of $\mathbf{1}$, an alternative $\mathrm{C} 6-\mathrm{C} 10$ cyclization leads to the core structure of mangicol A via a key 5-9-5 tricyclic intermediate (Supplementary Scheme S2).

In 2015, we proposed that the amino acid sequence of the terpene cyclase domain of bifunctional terpene synthases might reflect the first cyclization mode (Figure 3). ${ }^{9}$ A phylogenetic analysis showed that PaPS could be classified into clade $\mathrm{B}$, which catalyzes the formation of a 5-11 ring system via a C1-III-IV cyclization (type B cyclization). The elucidated cyclization mechanism of PaPS is consistent with our proposal. Furthermore, this has been extended to two more clade B-bifunctional terpene synthases, that is, astellifadiene synthase and stellatatriene synthase, which have recently been identified. ${ }^{19,20}$

In this study, we developed an efficient method to synthesize sitespecifically isotope-labeled oligoprenyl diphosphate precursors, such as DMAPP, GPP, FPP and GGPP, starting from four chemically synthesized $\left[{ }^{2} \mathrm{H}\right]$-IPPs by enzymatic transformation. By using these precursors, we demonstrated that the MS-based analysis of sitespecifically isotope-labeled terpenes provides sufficient information to elucidate the cyclization mechanism catalyzed by PaPS. During this study, we proposed a general strategy for the assignment of individual fragment ions: (1) information on the location of each isoprene unit (outline of fragmentation) could be obtained by analysis of the samples from the $\left[\mathrm{C}^{2} \mathrm{H}_{3}\right]$-labeled precursors; (2) detailed MS analysis of terpenes from isoprene unit-specifically labeled precursors provided useful information to assign the structure of the eliminated fragments; (3) fragmentation process of terpene could be proposed on the basis of chemical structure of cyclic terpene and labeling pattern of the eliminated fragments. In addition, overlapped ions with the same molecular m.u. could be separated by using a $\left[\mathrm{C}^{2} \mathrm{H}_{3}\right]$-labeled precursor and further analysis of 3 m.u.-shifted peaks provided additional information.

This highly sensitive method can be applied to a number of structurally complex terpenes. It was pointed out that a careful interpretation is required to perform the analysis due to the non-specific hydrogen migrations during the fragmentation processes and the kinetic isotope effects. ${ }^{21}$ These may be overcome by further confirmation of the labeling pattern by detailed HR-MS and MS/MS analyses, which were not used in this study. Recently, a systematic investigation into the EI-MS fragmentation mechanism of sesquiterpenes using all possible ${ }^{13} \mathrm{C}$-labeled isotopomers of FPP was also reported. ${ }^{21,22}$ Thus, several methods for investigating the cyclization mechanism of terpene synthases are now available. These mechanisms will contribute to the rational engineering of terpene synthases for the generation of diverse terpene skeletons.

\section{METHODS}

\section{General}

Oligonucleotides for polymerase chain reaction (PCR) were purchased from Hokkaido System Science Co., Ltd. Sequence analysis of the PCR fragment was performed by an automatic DNA sequencer (Applied Biosystems, Foster, CA, USA, ABI PRISM 310 Genetic Analyzer). Cell disruption was dealt with a ultrasonic disrupter UR-200P (TOMY SEIKO, Tokyo, Japan). Analysis of the 


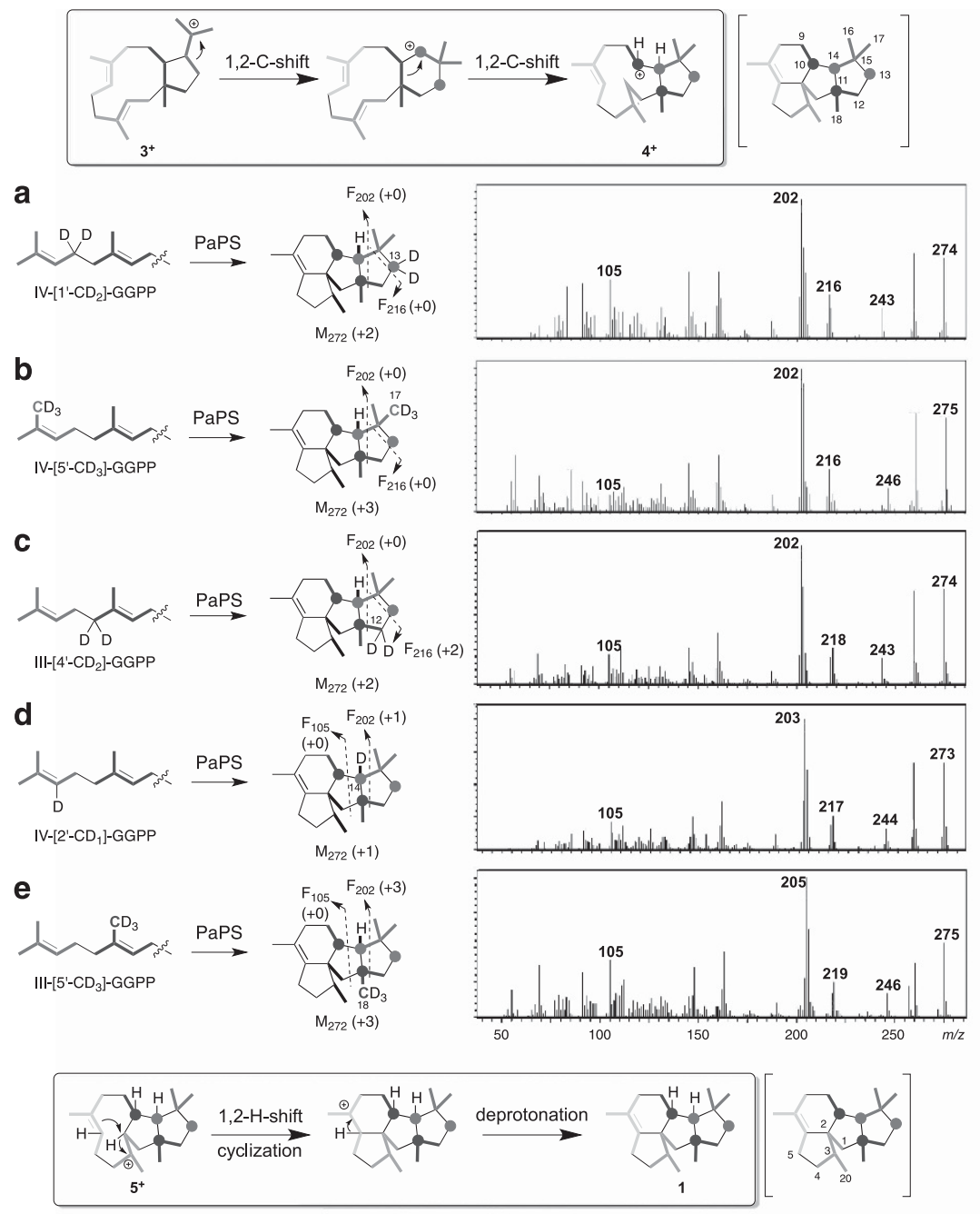

f
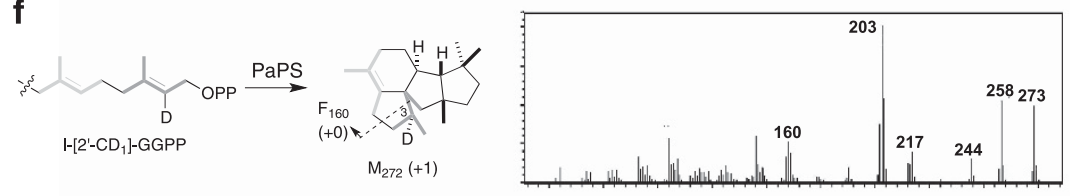

g
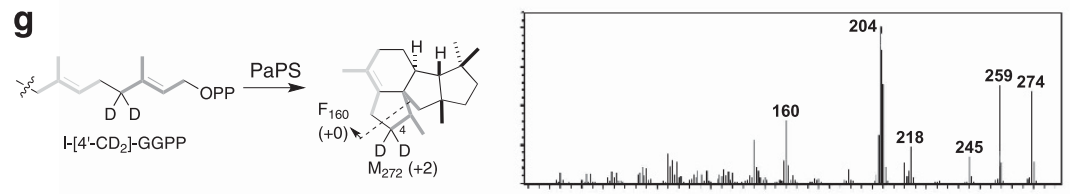

h
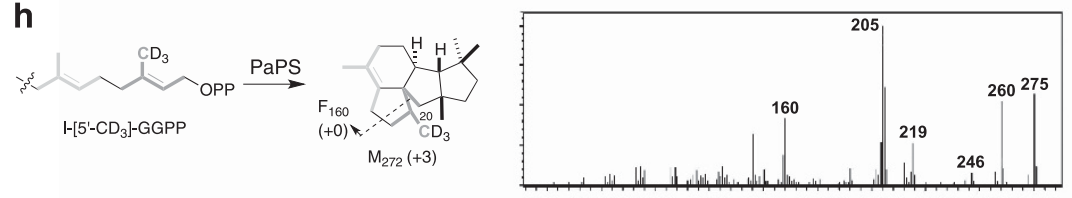

i
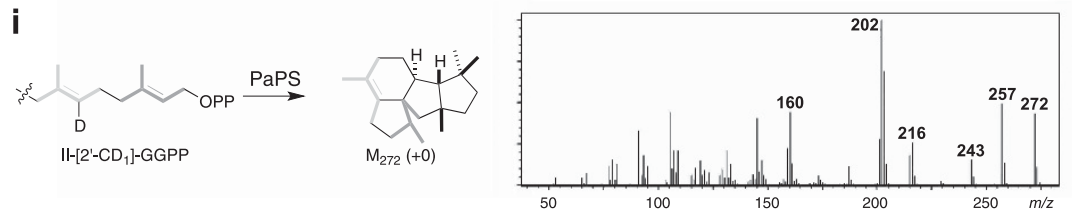

Figure 2 MS spectra of deuterium labeled 1; (a) IV-[1'-CD2]-GGPP, (b) IV-[5'-CD3]-GGPP, (c) III-[4'-CD2]-GGPP, (d) IV-[2'-CD1]-GGPP, (e) III-[5'-CD3]GGPP, (f) I-[2'-CD1]-GGPP, (g) I-[4'-CD2]-GGPP, (h) I-[5'-CD3]-GGPP and (i) II-[2'-CD1]-GGPP. The observed mass shifts are shown in the parenthesis. A full colour version of this figure is available at the Journal of Antibiotics journal online. 
Type A
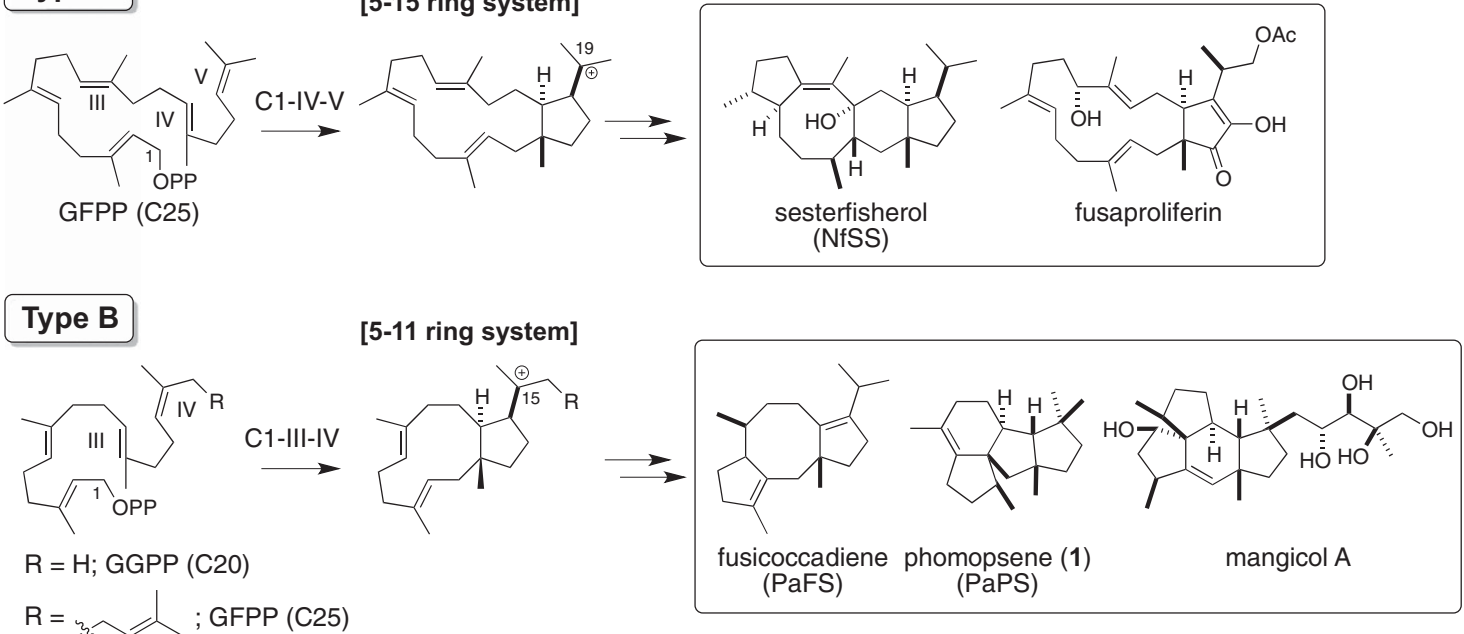

Figure 3 Classification of di/sesterterpenes based on the initial cyclization mode.

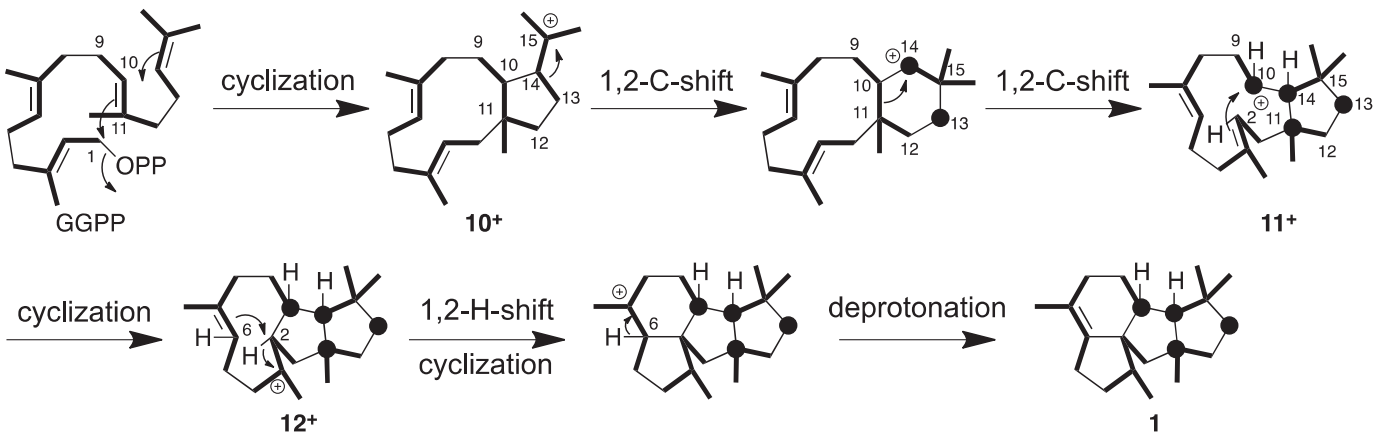

Scheme 2 Proposed cyclization mechanism of PaPS.

samples during protein purification was performed using SDS-polyacrylamide gel electrophoresis, and the proteins were visualized by using coomassie brilliant blue staining. Protein concentration was determined by the bradford method with bovine serum albumin as a standard.

\section{Strain and culture conditions}

Escherichia coli DH5 $\alpha$ were used for cloning and the following standard recombinant DNA techniques. E. coli BL21(DE3) was used for protein expression.

\section{Cloning and protein expression}

To generate the overexpression construct for idi and ispA, each gene was amplified using genomic DNA of E. coli as template and following primer sets; IDI-F (5'-GGGATTCCATATGCAAACGGAACACGTCATTTTAT-3' ${ }^{\prime}$ NdeI site underlined), IDI-R (5'-CCGGAATTCTTATTTAAGCTGGGTAAATGCAGA TA-3', EcoRI site underlined) for idi overexpression, IspA-F (5'-AGGATCCA TGGACTTTCCGCAGC-3', BamHI site underlined) and IspA-R (5'-CCC GAATTCTTATTTATTACGCTGGATG-3', EcoRI site underlined) for ispA overexpression. The PCR products were digested with appropriate restriction enzymes and cloned to the corresponding sites of pET28a to generate pET28aidi and pET28a-ispA.

Mutation of isp $A$ was introduced into pET28a-ispA by PCR using IspA-S81FF (5'-AGTAAGCGTGGATACACTCAACGG- $3^{\prime}$ ) and IspA-S81F-R (5'-TTTTAA TTCATGATGATTTACCGGCAA- $3^{\prime}$, mutation site underlined). The PCR product was then dealt with T4 Polynucleotide Kinase for phosphorylation of $5^{\prime}-\mathrm{OH}$ termini of the polynucleotides and re-ligated using Ligation high Ver.2 to generate pET28a-ispA-S81F.
pET28a-idi, pET28a-ispA and pET28a-ispA-S81F were separately introduced into E. coli BL21(DE3) for overexpression. Protein expression and purification was performed according to the literature procedure. ${ }^{15-17}$

\section{Enzyme synthesis of deuterium labeled-2}

Labeling of the first unit. Typical conditions are as follows; a reaction mixture (100 $\mu \mathrm{l}$ of Tris- $\mathrm{HCl}$ buffer (pH 7.4)) containing 1-2 $\mu \mathrm{g}$ of labeled-IPP, $5 \mu \mathrm{m}$ of $\mathrm{MgCl}_{2}, 0.5 \mathrm{~mm}$ of EDTA, $2 \mathrm{~mm}$ of DTT and 10-50 ng of IDI was incubated at $30^{\circ} \mathrm{C}$. After $3 \mathrm{~h}$, the reaction mixture was filtrated by utilizing the Amicon Ultra centrifugal filter (Merck Millipore, Billerica, MA, USA). To the filtrate was added 20-40 $\mu \mathrm{g}$ of non-labeled IPP, $1-5 \mathrm{ng}$ of IspA and 0.6-3 ng of PaPS was added and the reaction mixture was incubated at $30^{\circ} \mathrm{C}$. The reaction mixture was extracted with hexane and the crude extract was directly used for the analysis with MS-2010 (Shimadzu, Kyoto, Japan), using a DB-1 MS capillary column $(0.32 \mathrm{~mm} \times 30 \mathrm{~m}, 0.25 \mu \mathrm{m}$ film thickness; J\&W Scientific, Folsom, CA, USA).

Analytical conditions: the sample was injected into the column at $80^{\circ} \mathrm{C}$ in the splitless mode. After a $3 \mathrm{~min}$ isothermal hold at $80^{\circ} \mathrm{C}$, the column temperature was increased at $30^{\circ} \mathrm{C} \mathrm{min}{ }^{-1}$ to $290{ }^{\circ} \mathrm{C}$, with a 4 min isothermal hold at $290^{\circ} \mathrm{C}$. The flow rate of helium carrier gas was $0.66 \mathrm{ml} \mathrm{min}^{-1}$.

Labeling of the second or third unit. Typical conditions are as follows; a reaction mixture $(100 \mu \mathrm{l}$ of Tris- $\mathrm{HCl}$ buffer $(\mathrm{pH} 7.4))$ containing $1-2 \mu \mathrm{g}$ of non-labeled DMAPP, $2-4 \mu \mathrm{g}$ of labeled-IPP, $5 \mu \mathrm{M}$ of $\mathrm{MgCl}_{2}, 0.5 \mathrm{mM}$ of EDTA, $2 \mathrm{~mm}$ of DTT and $1.3-13 \mathrm{ng}$ of IspA-S81F was incubated at $30^{\circ} \mathrm{C}$. After $3 \mathrm{~h}$, the reaction mixture was filtrated by utilizing the Amicon Ultra $0.5 \mathrm{ml}$ centrifugal filter. To the filtrate was added $20-40 \mu$ g of non-labeled IPP, 
1-5 ng of IspA and $0.6-3 \mathrm{ng}$ of PaPS was added and the reaction mixture was incubated at $30^{\circ} \mathrm{C}$. The reaction mixture was extracted with hexane and the crude extract was directly analyzed by GC-MS as in the similar conditions described above. The similar reaction conditions with non-labeled GPP and IspA instead of DMAPP and IspA-S81F was applied for labeling of FPP on the third unit. The labeled FPP was used for the PaPS reaction.

Labeling of the fourth unit. Typical conditions are as follows; a reaction mixture (100 $\mu \mathrm{l}$ of Tris- $\mathrm{HCl}$ buffer ( $\mathrm{pH}$ 7.4)) containing 1-3 $\mu$ g of non-labeled FPP, $4 \mu \mathrm{g}$ of the labeled-IPP, $5 \mu \mathrm{M}$ of $\mathrm{MgCl}_{2}, 0.5 \mathrm{~mm}$ of EDTA, $2 \mathrm{~mm}$ of DTT and $0.6-3$ ng of PaPS was incubated at $30^{\circ} \mathrm{C}$. The reaction mixture was extracted with hexane and the crude extract was directly analyzed by GC-MS as in the similar conditions described above.

\section{CONFLICT OF INTEREST}

The authors declare no conflict of interest.

\section{ACKNOWLEDGEMENTS}

This work was financially supported by Grants-in-Aid for Scientific Research from the Ministry of Education, Culture, Sports, Science and Technology, Japan (JSPS KAKENHI Grant Number JP15H01835 (HO), JP16H03277 (AM), JP16H06446 (AM) and JP10F00032 (JSPS PD-fellowship to SS).

1 Dewick, P. M. The biosynthesis of $\mathrm{C}_{5}-\mathrm{C}_{25}$ terpenoid compounds. Nat. Prod. Rep. 19, 181-222 (2002).

2 Christianson, D. W. Structural biology and chemistry of the terpenoid cyclases. Chem. Rev. 106, 3412-3442 (2006).

3 Tantillo, D. J. Biosynthesis via carbocations: theoretical studies on terpene formation. Nat. Prod. Rep. 28, 1035-1053 (2011).

4 Cane, D. E. Enzymatic formation of sesquiterpenes. Chem. Rev. 90, 1089-1103 (1990)

5 Ernst, M., Silva, D. B., Silva, R. R., Vencio, R. Z. N. \& Lopes, P. Mass spectrometry in plant metabolomics strategies: from analytical platforms to data acquisition and processing. Nat. Prod. Rep. 31, 784-806 (2014).

6 Toyomasu, T. et al. Fusicoccins are biosynthesized by an unusual chimera diterpene synthase in fungi. Proc. Natl Acad. Sci. USA 104, 3084-3088 (2007).
7 Toyomasu, T. et al. Biosynthetic gene-based secondary metabolite screening: a new diterpene, methyl phomopsenonate, from the fungus Phomopsis amygdali. J. Org. Chem. 74, 1541-1548 (2009).

8 Chiba, R., Minami, A., Gomi, K. \& Oikawa, H. Identification of ophiobolin F synthase by a genome mining approach: a sesterterpene synthase from Aspergillus clavatus. Org. Lett. 15, 594-597 (2013).

9 Ye, Y. et al. Genome mining for sesterterpenes using bifunctional terpene synthases reveals a unified intermediate of di/sesterterpenes. J. Am. Chem. Soc. 137, 11846-11853 (2015).

10 Okada, M. et al. Genome-based discovery of an unprecedented cyclization mode in fungal sesterterpenoid biosynthesis. J. Am. Chem. Soc. 138, 10011-10018 (2016).

11 Peterson, D. J. A carbonyl olefination reaction using silyl-substituted organometallic compounds. J. Org. Chem. 33, 780-784 (1968).

$12 \mathrm{Ma}, \mathrm{S}$. \& Negishi, E. Anti-carbometalation of homopropargyl alcohols and their higher homologues via non-chelation-controlled syn-carbometalation and chelation-controlled isomerization. J. Org. Chem. 62, 784-785 (1997).

13 Davisson, V. J. et al. Phosphorylation of isoprenoid alcohols. J. Org. Chem. 51, 4768-4779 (1986).

14 Scholte, A. A. \& Vederas, J. C. Incorporation of deuterium-labelled analogs of isopentenyl diphosphate for the elucidation of the stereochemistry of rubber biosynthesis. Org. Biomol. Chem. 4, 730-742 (2006).

15 Hahn, F. M., Hurlburt, A. P. \& Poulter, C. D. Escherichia coli open reading frame 696 is idi, a nonessential gene encoding isopentenyl diphosphate isomerase. J. Bacteriol. 181, 4499-4504 (1999)

16 Reiling, K. K. et al. Mono and diterpene production in Escherichia coli. Biotechnol. Bioeng. 87, 200-212 (2004).

$17 \mathrm{Ku}$, B., Jeong, J.-C., Mijts, B. N., Schmidt-Dannert, C. \& Dordick, J. S. Preparation, characterization, and optimization of an in vitro C30 carotenoid pathway. Appl. Environ. Microbiol. 71, 6578-6583 (2005)

18 Renner, M. K., Jensen, P. R. \& Fenical, W. Mangicols: structures and biosynthesis of a new class of sesterterpene polyols from a marine fungus of the genus Fusarium. J. Org. Chem. 65, 4843-4852 (2000).

19 Matsuda, Y.. Mitsuhashi, T., Quan, Z. \& Abe, I. Molecular basis for stellatic acid biosynthesis: a genome mining approach for discovery of sesterterpene synthases. Org. Lett. 17, 4644-4647 (2015).

20 Matsuda, Y. et al. Astellifadiene: structure determination by NMR spectroscopy and crystalline sponge method, and elucidation of its biosynthesis. Angew. Chem. Int. Ed. 55, 5785-5788 (2016)

21 Rabe, P. \& Dichschat, J. S. The EIMS fragmentation mechanisms of the sesquiterpenes corvol ethers A and B, epi-cubebol and isodauc-8-en-11-ol. Beilstein J. Org. Chem. 12, 1380-1394 (2016)

22 Rabe, P., Klapschinski, T. A. \& Dickschat, J. S. Position-specific mass shift analysis: a systematic method for investigating the El-MS fragmentation mechanism of epi-isozizaene. ChemBioChem. 17, 1333-1337 (2016).

Supplementary Information accompanies the paper on The Journal of Antibiotics website (http://www.nature.com/ja) 